\title{
Mandatory Seat Belt Laws in Eight States: A Time-Series Evaluation
}

\author{
Alexander C. Wagenaar, Richard G. Maybec, and Kathlecn P. Sullivan
}

\begin{abstract}
We cxamined state-specific and aggregate effects of U.S. legislation requiring the use of seat belts among front-seat motor vehicle occupants. Effects of compulsory seat belt use on the number of occupants fatally injured in traffic crashes were examined in the first eight states adopting such laws. Monthly data on crash fatalities between January 1976 and June 1986 were analyzed using Box-Tiao intervention analysis time-series methods. Because the new laws apply only to front-seat occupants, front-seat occupant fatalities were compared with: (1) rear-seat fatalities; (2) nonoccupant fatalities (motorcyclists, pedalcyclists, pedestrians); and (3) fatalities among front-seat occupants in neighboring states without compulsory seat belt use. Exposure to risk of crash involvement was controlled by analyzing fatality rates per vehicle mile traveled. Results revealed a statistically significant decline of $8.7 \%$ in the rate of front-seat fatalities in the first eight states with seat belt laws. The fatality rate declined $9.9 \%$ in states with primary enforcement laws and $6.8 \%$ in states with secondary enforcement only. Rates of rear-seat and nonoccupant fatalities did not change when the belt laws were implemented.
\end{abstract}

Use of automobile safety belts reduces the probability of death in a motor vehicle crash by $30 \%$ to $50 \%$ (Evans, 1986; O'Day \& Flora, 1982). To increase belt use, laws requiring their use were first implemented in Aus-

\footnotetext{
Alexander C. Wagenaar, $\mathrm{PhD}$, is an associate research scientist, Richard G. Maybee, PhD, is a research associate, and Kathleen $P$. Sullivan, $M A$, is a research assistant in the Injury Analysis and Prevention Group at the University of Michigan Transportation Research Institute, 2901 Baxter Hoad, Ann Arbor, MI 48109-2150.

An earlier version of this paper was presented at the 11th International Technical Conference on Experimental Safety Vehicles, Washington, DC, May 12-15, 1987.
}

tralia in 1971 and spread to a number of European countries, Canadian provinces, and other jurisdictions in the subsequent decade. In the mid-1980s, selected states in the United States implemented compulsory belt use laws. The objective of this study was to evaluate the effects of belt laws on motor vehicle fatality rates in the first eight U.S. states implementing such laws.

This research was supported in part by a gift from the United States Motor Vehicle Manufacturers Association to the University of Michigan Transportation Research Institute. The assistance of Charles P. Compton with data file construction is gratefully acknowledged. 
Numerous studies have found increased belt use and reduced rates of traffic fatalities following implementation of compulsory belt use laws. Although effects varied, rates of seat belt use have typically doubled or tripled immediately after belt laws took effect, both in the United States (Table 1) and in other countries (Table 2). After immediate dramatic increases in belt use at the time belt laws first took effect, many jurisdictions experienced some decay in use over the subsequent months or years (Campbell, Stewart, \& Campbell, 1986). Estimated fatality reductions following implementation of compulsory belt use vary widely from country to country (from 0 to $80 \%$; Table 3 ). Within the United States, preliminary estimates of the effect of belt laws on fatalities cluster much more narrowly in the range of $1 \%$ to $20 \%$ (Table 4 ). Many of these studies, especially the earlier ones, used nonrandom samples, inadequate control groups, and unreported analytic methods.

\section{METHOD}

\section{Research Design}

We evaluated eight U.S. states that implemented mandatory seat belt use laws prior to October 1985, using monthly data on traffic fatalities from January 1976 through June 1986. A longitudinal or time-series design was used to ensure that observed changes in fatalities were not due to longterm cycles or trends or were not a result of a regression-to-the-mean effect. In the absence of random assignment, time-series de signs with comparison groups have the highest possible levels of internal validity (Cook \& Campbell, 1979).

To further strengthen causal inferences concerning the relationship between compulsory seat belt laws and traffic fatalities, we examined two types of control groups that one would not expect to be affected by the new laws. First, we paired each "experimental" state that recently implemented a seat belt law with a neighboring "control" state that did not implement a belt law during the period under study. States analyzed include: New York with a belt law versus
Pennsylvania without, New Jersey versus Maryland, Michigan versus Ohio, Illinois versus Indiana, Texas versus Georgia, Nebraska versus Kansas, Missouri versus Tennessee, and North Carolina versus Virginia. ${ }^{1}$ Second, within the experimental states we examined two categories of traffic fatalities not directly affected by the new laws - rearseat occupants and nonoccupants (including pedestrians, motorcyclists, and pedalcyclists).

\section{Data Collection}

All fatality data were based on the Fatal Accident Reporting System (FARS) maintained by the U.S. National Highway Traffic Safety Administration (NHTSA). Monthly counts of the number of fatalities were calculated separately within each state for front-seat occupants, rear-seat occupants, and nonoccupants. Occupant fatalities included only those traveling in passenger cars, vans, light trucks, and utility vehicles. Medium and heavy trucks, buses, and a variety of special vehicles were excluded because some are exempted from provisions of the seat belt laws and others were covered by preexisting regulations requiring seat belt use. All analyses were limited to persons age 10 and over because compulsory restraint use laws for young children were implemented several years before the adult seat belt laws took effect. Although most child restraint laws are limited to those under age 4 , spill-over effects on older children have been reported (Wagenaar \& Webster, 1986). The length of the resulting time series varied from 107 baseline months in New York to 117 baseline months in North Carolina, and from 9 postlaw months in North Carolina to 19 postlaw months in New York.

Exposure to risk of crash involvement was controlled by dividing all of the fatality frequency time-series by the number of vehicle miles traveled (VM'l') within each of the states under study. The resulting rates of fatalities per VMT were used in all subsequent

\footnotetext{
ITwo of the comparison states, Ohio and Tennessee, implemented compulsory belt use laws in the spring of 1986. Analyses involving these states were limited to the period in which no belt law was in effect.
} 
TABLE 1

EFFECTS OF U.S. SEAT BELT LAWS ON RESTRAINT USE

\begin{tabular}{|c|c|c|c|c|}
\hline Jurisdiction & $\begin{array}{l}\text { Effective } \\
\text { Month }\end{array}$ & $\begin{array}{l}\text { Month } \\
\text { Observed }\end{array}$ & $\begin{array}{l}\text { Use } \\
\text { Rate }\end{array}$ & Source \\
\hline New York & $12 / 84$ & $\begin{array}{r}10 / 84 \\
4 / 85 \\
9 / 85\end{array}$ & $\begin{array}{l}16 \% \\
57 \% \\
46 \%\end{array}$ & Rood, Kraichy, \& Carubia (1985) \\
\hline & & $\begin{array}{l}1 / 85 \\
4 / 85 \\
2 / 86\end{array}$ & $\begin{array}{l}69 \% \\
60 \% \\
44 \%\end{array}$ & Williams, Wells, Lund (1986) \\
\hline & & $\begin{array}{r}4 / 85 \\
487 / 86\end{array}$ & $\begin{array}{l}638 \\
378\end{array}$ & Pace, Thailer, \&wiatkowski (1986) \\
\hline New Jersey & $3 / 85$ & $7 / 85$ & $\begin{array}{l}18 \% \\
40 \%\end{array}$ & Brick et al. (cited in Williams et al., 1986) \\
\hline & & $\begin{array}{r}11 / 84 \\
3 / 85 \\
7 / 85 \\
4 / 86\end{array}$ & $\begin{array}{l}16 \% \\
51 \% \\
44 \% \\
38 \%\end{array}$ & Williams et al. (1986) \\
\hline Illinois & $7 / 85$ & $\begin{array}{r}4 / 85 \\
7 / 85 \\
12 / 85 \\
1 / 86 \\
3 / 86 \\
6 / 86\end{array}$ & $\begin{array}{l}16 \% \\
40 \% \\
35 \% \\
29 \% \\
32 \% \\
34 \%\end{array}$ & Mortimer (1986) \\
\hline Mi chi gan & $7 / 85$ & $\begin{array}{r}12 / 84 \\
4 / 85 \\
7 / 85 \\
12 / 85 \\
4 / 86 \\
7 / 86 \\
12 / 86\end{array}$ & $\begin{array}{l}18 \% \\
25 \% \\
61 \% \\
44 \% \\
44 \% \\
47 \% \\
44 \%\end{array}$ & Wagenaar, Molnar, Businski (1987) \\
\hline Texas & $9 / 85$ & $1-6 / 85$ & $\begin{array}{l}15 \% \\
66 \%\end{array}$ & $\begin{array}{l}\text { Hatfield, Hinshaw, Bunch, \& Bremer (1985) } \\
\text { Bunch, Hatfield, Hinshaw, \& Womack (1986) }\end{array}$ \\
\hline & & $3 / 86$ & 75\% & Dept. of Highways (cited in Campbell et at.. 1986) \\
\hline Nebraska & $9 / 85$ & $\begin{array}{r}11 / 85 \\
11 / 85 \\
2 / 86\end{array}$ & $\begin{array}{l}26 * \\
44 \% \\
38 \%\end{array}$ & $\begin{array}{l}\text { Office of Highway Safety } \\
\text { (clted in Campbell et al.., 1986) }\end{array}$ \\
\hline & & $2 / 87$ & $29 \%$ & Insurance Institute for Hi ghway Safety (IIHS)(1987b) \\
\hline Missouri & $10 / 85$ & $\begin{array}{r}7 / 85 \\
10 / 85\end{array}$ & $\begin{array}{l}12 \% \\
198\end{array}$ & Missouri Safety Center (cited in Campbel1 et a1.,1986) \\
\hline North Carolina & $10 / 85$ & $\begin{array}{r}9 / 85 \\
11 / 85 \\
1 / 86 \\
3 / 86 \\
5 / 86\end{array}$ & $\begin{array}{l}25 \% \\
44 \% \\
42 \% \\
45 \% \\
48 \%\end{array}$ & Campbe11. Stewart. Campbell (1986) \\
\hline & & $2 / 87$ & $78 x$ & IHHS (1987a) \\
\hline
\end{tabular}

Drivers oniy. 
TABLE 2

EFFECTS OF NON-U.S. SEAT BELT LAWS ON RESTRAINT USE

\begin{tabular}{|c|c|c|c|c|c|}
\hline Jurisdiction & $\begin{array}{l}\text { Effective } \\
\text { Month }\end{array}$ & $\begin{array}{l}\text { Period } \\
\text { Observed }\end{array}$ & $\begin{array}{l}\text { Use } \\
\text { Rate }\end{array}$ & Caments $^{a}$ & Source \\
\hline \multicolumn{6}{|l|}{ Australia } \\
\hline \multirow[t]{9}{*}{ Victoria } & $12 / 70$ & $5 / 71$ & $32 \%-48 \%$ & Front-seat occupants & Vul can (1977) \\
\hline & & $2 / 72$ & $47 \%-60 \%$ & & \\
\hline & & $2 / 73$ & $52 \%-65 \%$ & & \\
\hline & & $2 / 74$ & $67 x-79 x$ & & \\
\hline & & $2 / 75$ & $73 x-79 x$ & & \\
\hline & & $2 / 76$ & $73 \%-88 \%$ & & \\
\hline & & prelaw & $18 \%$ & Observation date not cfted & Joubert (1979) \\
\hline & & postlaw & $64 \%$ & Rural & \\
\hline & & postlaw & $75 \%$ & Urban & \\
\hline \multirow[t]{2}{*}{ Melbourne } & $12 / 70$ & $11 / 82$ & $95 \%$ & Drivers & Manders (1984) \\
\hline & & $3 / 84$ & $96 x$ & Drivers & \\
\hline \multirow{11}{*}{$\begin{array}{l}\text { Adelaide, } \\
\text { South Australia }\end{array}$} & $12 / 71$ & $10 / 71$ & $23 \%$ & Occupants with belts & Johinke (1977) \\
\hline & & $10 / 72$ & $78 \%$ & available & \\
\hline & & $10 / 75$ & $66 \%$ & & \\
\hline & & $\operatorname{mid} 76$ & $84 \%$ & & \\
\hline & & $10 / 64$ & $8 \%$ & All seating positions & Crinion, Foldvary, \\
\hline & & $10 / 70$ & $14 \%$ & & Lane (1975) \\
\hline & & $10 / 71$ & $23 x$ & & \\
\hline & & $10 / 72$ & $78 \%$ & & \\
\hline & & $11 / 82$ & $91 \%$ & Drivers & Road Traffic Board (1983) \\
\hline & & $11 / 82$ & $85 \%$ & Front-seat passengers & \\
\hline & & $11 / 82$ & $61 \%$ & Rear-seat passengers & \\
\hline Queen sl and & $1 / 72$ & $12 / 72$ & $90 \%$ & & Seeney (1977) \\
\hline \multirow{9}{*}{$\begin{array}{l}\text { New South Wales- } \\
\text { Sydney Metro }\end{array}$} & $10 / 71$ & $4 / 71$ & $30 \%$ & Drivers & Schnerring (1983) \\
\hline & & $10 / 71$ & $60 \%$ & Drivers & \\
\hline & & $11 / 71$ & $76 \%$ & Drivers & \\
\hline & & $12 / 72$ & $89 \%$ & Drivers & \\
\hline & & $12 / 73$ & $91 \%$ & Drivers & \\
\hline & & $7 / 75$ & $91 \%$ & Drivers & \\
\hline & & $7 / 77$ & $91 \%$ & Drivers & \\
\hline & & $7 / 79$ & $89 \%$ & Drivers & \\
\hline & & $7 / 81$ & $84 \%$ & Drivers & \\
\hline \multirow[t]{8}{*}{ Austria } & $7 / 76$ & prelaw & $5 \%-10 \%$ & Urban & Marburger (1986) \\
\hline & & prelaw & $20 \%-25 \%$ & Rural & \\
\hline & & postlaw & $10 \%-15 \%$ & Urban & \\
\hline & & postlaw & $40 \%$ & Urban & \\
\hline & & $9 / 84$ & $81 \%$ & Urban & \\
\hline & & $9 / 84$ & $82 \%$ & Rural & \\
\hline & & $8 / 85$ & $81 \%$ & Urban & \\
\hline & & $8 / 85$ & $82 \%$ & Rural & \\
\hline \multirow[t]{4}{*}{ Bel gium } & $6 / 75$ & prelaw & $17 \%$ & & Fi sher $(1980)$ \\
\hline & & postlaw & $87 \%$ & & \\
\hline & & $11 / 84$ & $70 x$ & Rural & Marburger (1986) \\
\hline & & $11 / 84$ & $60 x$ & Urban & \\
\hline
\end{tabular}


TABLE 2

(CONTINUED)

\begin{tabular}{|c|c|c|c|c|c|}
\hline Juri sdiction & $\begin{array}{l}\text { Effective } \\
\text { Nonth }\end{array}$ & $\begin{array}{l}\text { Pertiod } \\
\text { Observed }\end{array}$ & $\begin{array}{l}\text { Use } \\
\text { Rate }\end{array}$ & Coments ${ }^{a}$ & Source \\
\hline \multicolumn{6}{|l|}{ Canada } \\
\hline \multirow[t]{4}{*}{ British Col umbia } & $10 / 77$ & $\begin{array}{l}\text { prelaw } \\
\text { postlaw }\end{array}$ & $\begin{array}{l}20 \%-24 \% \\
50 \%\end{array}$ & & $\begin{array}{l}\text { Rockerbie (personal com- } \\
\text { munication, } 2 / 16 / 83 \text { ) }\end{array}$ \\
\hline & & $4 / 83$ & $55 \%$ & & British Columbia \\
\hline & & $6 / 83$ & $67 \%$ & & Research (1983) \\
\hline & & $11 / 83$ & $67 \%$ & & Arora (1985) \\
\hline \multirow[t]{2}{*}{ New Brunswi ck } & $9 / 83$ & $\begin{array}{l}11 / 82 \\
11 / 83\end{array}$ & $\begin{array}{r}4 \% \\
66 \%\end{array}$ & $\begin{array}{l}\text { Drivers } \\
\text { Drivers }\end{array}$ & Arora (1985) \\
\hline & & $8 / 84$ & $73 \%$ & & $\begin{array}{l}\text { New Brunswick Dept. of } \\
\text { Tran sportatt on }(1984)\end{array}$ \\
\hline \multirow[t]{5}{*}{ Newf ound $l$ and } & $7 / 82$ & $11 / 81$ & $9 \%$ & & Arora (1982) \\
\hline & & $11 / 82$ & $68 \%$ & & \\
\hline & & $11 / 83$ & $76 \%$ & Urban & Arora (1985) \\
\hline & & $11 / 83$ & $61 \%$ & Rural & \\
\hline & & $7 / 84$ & $74 \%$ & & Murray (1984) \\
\hline \multirow[t]{3}{*}{ Mani toba } & $1 / 84$ & $11 / 83$ & $11 \%$ & & Arora (1985) \\
\hline & & $11 / 84$ & $62 x$ & & \\
\hline & & $6 / 84$ & 798 & Drivers & DataCon (1984) \\
\hline \multirow[t]{9}{*}{ Ontario } & $1 / 76$ & $12 / 75$ & $21 \%$ & & Snow (1979) \\
\hline & & $2 / 76$ & $77 \%$ & & \\
\hline & & $6 / 76$ & $50 \%$ & & \\
\hline & & $5 / 77$ & $50 \%$ & Increased enforcement & Pierce (1979) \\
\hline & & $5 / 78$ & $65 \%$ & $\operatorname{mid} 177$ & \\
\hline & & $9-12 / 80$ & $49 \%$ & & Matthews (1982) \\
\hline & & $11 / 82$ & $49 \%$ & & Arora (1985) \\
\hline & & $11 / 83$ & $60 \%$ & & \\
\hline & & $5 / 84$ & $70 \%$ & & Jonah \& Lawson (1986) \\
\hline \multirow[t]{4}{*}{ Montreal } & $8 / 76$ & $5-6 / 76$ & $15 \%$ & Drivers & Stul ginskas \& Pless \\
\hline & & $5-6 / 77$ & $33 x$ & Drivers & $(1983)$ \\
\hline & & $5-6 / 78$ & $45 \%$ & Drivers & \\
\hline & & $5-6 / 81$ & $56 \%$ & Drivers & \\
\hline \multirow[t]{3}{*}{ Quebec } & $8 / 76$ & $11 / 82$ & $68 \%$ & Drivers & Arora (1985) \\
\hline & & $11 / 83$ & $60 \%$ & Drivers & \\
\hline & & $6 / 83$ & $\begin{array}{l}60 \% \\
74 \%\end{array}$ & $\begin{array}{l}\text { Urban drivers } \\
\text { Freeway drivers }\end{array}$ & $\begin{array}{l}\text { Regle de } 1 \text { 'assurance Au- } \\
\text { tomobtle du Quebec (cited } \\
\text { in Jonah \& Lawson, 1986) }\end{array}$ \\
\hline
\end{tabular}


TABLE 2

(CONTINUED)

\begin{tabular}{|c|c|c|c|c|c|}
\hline Juri sdiction & $\begin{array}{l}\text { Effective } \\
\text { Month }\end{array}$ & $\begin{array}{l}\text { Period } \\
\text { Observed }\end{array}$ & $\begin{array}{l}\text { Use } \\
\text { Rate }\end{array}$ & Coments ${ }^{a}$ & Source \\
\hline \multicolumn{6}{|l|}{ Canada (cont.) } \\
\hline \multirow[t]{14}{*}{ Saskatchewan } & $7 / 77$ & prelaw & $26 \%$ & Drivers & Simpson \& Warren (1981) \\
\hline & & postlaw & $78 \%$ & Drivers & \\
\hline & & $5 / 77$ & $24 \%$ & & Shiels (1978) \\
\hline & & $7 / 77$ & $65 \%$ & & \\
\hline & & $10 / 77$ & 738 & & \\
\hline & & $5 / 78$ & $60 \%$ & & \\
\hline & & $7 / 77$ & $52 \%$ & Drivers & Bergen. Watson, Rivett, \\
\hline & & $10 / 77$ & 708 & Drivers & \& Shiels (1979) \\
\hline & & $5 / 78$ & $55 \%$ & Drivers & \\
\hline & & $5 / 79$ & $70 \%$ & Drivers & \\
\hline & & $11 / 80$ & $56 \%$ & Drivers & Arora $(1982,1985)$ \\
\hline & & $11 / 81$ & $49 \%$ & Drivers & \\
\hline & & $11 / 82$ & $48 \%$ & Drivers & \\
\hline & & $11 / 83$ & $54 \%$ & Drivers & \\
\hline \multirow[t]{2}{*}{ Denmark } & $1 / 76$ & prelaw & $19 \%$ & & Marburger (1986) \\
\hline & & postlaw & $74 \%$ & & \\
\hline \multirow[t]{3}{*}{ England } & $2 / 83$ & $1 / 83$ & $43 \%$ & & Ashton, Mackay, Camm \\
\hline & & $5 / 83$ & $95 \%$ & & $(1983)$ \\
\hline & & $2 / 83$ & $90 \%$ & & Mackay $(1984 a, 1984 b)$ \\
\hline \multirow[t]{17}{*}{ Finland } & $7 / 75$ & prelaw & $15 *-20 *$ & & Oranen (1977) \\
\hline & & $6 / 75$ & $30 \%$ & Hi ghway & \\
\hline & & $6 / 75$ & 96 & Urban & \\
\hline & & $8 / 75$ & $68 \%$ & Hi ghway & \\
\hline & & $8 / 75$ & 53* & Urban & \\
\hline & & $7 / 76$ & 64\% & Hi ghway & \\
\hline & & $8 / 76$ & $37 \mathrm{t}$ & Urban & \\
\hline & & prelav & $8 *$ & Urban & Berard-Anderson (cited in \\
\hline & & prelaw & $31 \%$ & Rural & Fi sher, 1980 ) \\
\hline & & postlaw & $38 \%$ & Urban & \\
\hline & & postlaw & $66 \%$ & Rural & \\
\hline & & $8-9 / 78$ & $71 \%$ & Hi ghways & Oranen Kotvurova (1981) \\
\hline & & $8-9 / 78$ & $41 \%$ & Urban & \\
\hline & & $4 / 82$ & $87 \%$ & Urban & Marburger (1986) \\
\hline & & $4 / 82$ & $86 \%$ & Rural & \\
\hline & & $9 / 83$ & $82 \%$ & Urban & \\
\hline & & $9 / 83$ & $92 \%$ & Rural & \\
\hline \multirow[t]{3}{*}{ France } & $7 / 73$ & prelaw & $20 \%-25 \%$ & & Fisher (1980) \\
\hline & & $7 / 73$ & $80 \%$ & & \\
\hline & & $10 / 73$ & $50 \%$ & & \\
\hline
\end{tabular}


TABLE 2

(CONTINUED;

\begin{tabular}{|c|c|c|c|c|c|}
\hline Jurf sdiction & $\begin{array}{l}\text { Effective } \\
\text { Month }\end{array}$ & $\begin{array}{l}\text { Perlod } \\
\text { Coserved }\end{array}$ & $\begin{array}{l}\text { Use } \\
\text { Rate }\end{array}$ & coments ${ }^{a}$ & Source \\
\hline France (cont.) & $7 / 73$ & $\begin{array}{l}1972 \\
1973 \\
1974 \\
1975\end{array}$ & $\begin{array}{l}20 \% \\
26 \% \\
67 \% \\
80 \%\end{array}$ & & $\begin{array}{l}\text { Chodkiewicz \& Dubarry } \\
\text { (1977) }\end{array}$ \\
\hline & & $\begin{array}{l}7 / 73 \\
11 / 73 \\
\text { early } ' 74 \\
1979\end{array}$ & $\begin{array}{l}80 \% \\
50 \% \\
80 \% \\
70 \%-75 \%\end{array}$ & Law applied to rural only & Gerondeau (1979 \\
\hline & & $\begin{array}{l}1974 \\
1975 \\
1976 \\
1977 \\
1978 \\
1979 \\
1980\end{array}$ & $\begin{array}{l}54 \% \\
76 \% \\
79 \% \\
72 \% \\
67 \% \\
69 \% \\
79 \%\end{array}$ & $\begin{array}{l}\text { Law expanded to all roads } \\
\text { in } 1979\end{array}$ & Gerondeau (1981) \\
\hline & & $\begin{array}{l}1982 \\
1982\end{array}$ & $\begin{array}{l}95 \% \\
75 \%\end{array}$ & $\begin{array}{l}\text { Hi ghways } \\
\text { Other nonurban }\end{array}$ & Hartemann et al. (1984) \\
\hline Ireland & $2 / 79$ & $\begin{array}{l}\text { Fall '78 } \\
\text { Fall '78 } \\
\text { Summer '79 } \\
\text { Summer '79 }\end{array}$ & $\begin{array}{r}19 \% \\
9 \% \\
46 \% \\
38 \%\end{array}$ & $\begin{array}{l}\text { Drivers on national roads } \\
\text { Drivers on other roads } \\
\text { Drivers on national roads } \\
\text { Drivers on national roads }\end{array}$ & Heame (1981) \\
\hline Israel & $7 / 75$ & $\begin{array}{l}\text { prelaw } \\
8 / 75 \\
1976 \\
1977\end{array}$ & $\begin{array}{r}6 \% \\
77 \% \\
83 \% \\
70 \%\end{array}$ & & $\begin{array}{l}\text { Hakkert, Zaidel, } \\
\text { Sarelle (1981) }\end{array}$ \\
\hline Netherlands & $6 / 75$ & $\begin{array}{l}1974 \\
1974 \\
7 / 76 \\
7 / 76\end{array}$ & $\begin{array}{l}11 \% \\
24 \% \\
58 \% \\
75 \%\end{array}$ & $\begin{array}{l}\text { Urban } \\
\text { Rural } \\
\text { Urban } \\
\text { Rural }\end{array}$ & Fi sher (1980) \\
\hline & & $\begin{array}{l}1983 \\
1983\end{array}$ & $\begin{array}{l}46 \% \\
65 \%\end{array}$ & $\begin{array}{l}\text { Urban } \\
\text { Rural }\end{array}$ & Vaaje (1986) \\
\hline New Zeal and & $6 / 72$ & $\begin{array}{l}5 / 72 \\
6 / 72 \\
1974 \\
1975\end{array}$ & $\begin{array}{l}40 \% \\
87 \% \\
83 \% \\
89 \%\end{array}$ & & Toomath (1977) \\
\hline Norway & $9 / 75$ & $\begin{array}{c}\text { prelaw } \\
\text { prelaw } \\
1976 \\
1976 \\
1977 \\
1977\end{array}$ & $\begin{array}{l}15 \% \\
37 \% \\
28 \% \\
59 \% \\
30 \% \\
63 \%\end{array}$ & $\begin{array}{l}\text { Urban } \\
\text { Rural } \\
\text { Urban } \\
\text { Rural } \\
\text { Urban } \\
\text { Rural }\end{array}$ & Fisher (1980) \\
\hline & & $\begin{array}{l}3 / 80 \\
3 / 80\end{array}$ & $\begin{array}{l}74 \% \\
90 \%\end{array}$ & $\begin{array}{l}\text { Urban } \\
\text { Rural }\end{array}$ & Oranen \& Koivurova (1981) \\
\hline
\end{tabular}




\begin{tabular}{|c|c|c|c|c|c|}
\hline Jurisdiction & $\begin{array}{l}\text { Effective } \\
\text { Month }\end{array}$ & $\begin{array}{l}\text { Period } \\
\text { Observed }\end{array}$ & $\begin{array}{l}\text { Use } \\
\text { Rate }\end{array}$ & Coments $^{a}$ & Source \\
\hline \multirow[t]{5}{*}{ Puerto Rico } & $1 / 74$ & $7 / 73$ & $5 \%$ & & Fi sher $(1980)$ \\
\hline & & $5 / 74$ & $24 \%$ & & \\
\hline & & $9 / 74$ & $7 \%$ & & \\
\hline & & $1 / 76$ & $34 \%$ & & \\
\hline & & $5 / 77$ & $14 \%$ & & \\
\hline \multirow[t]{3}{*}{ South Africa } & $12 / 77$ & $11 / 77$ & $18 \%$ & & Fernie (1980) \\
\hline & & $3 / 78$ & $62 \%$ & & \\
\hline & & $9 / 79$ & $70 \%$ & & \\
\hline \multirow[t]{6}{*}{ Smeden } & $1 / 75$ & 1974 & $35 \%$ & & Bohl in (1973) \\
\hline & & 1975 & $84 \%$ & & \\
\hline & & 1974 & $36 \%$ & & Tingvall (ctted in \\
\hline & & 1978 & $79 \%$ & Urban & Fisher, 1980) \\
\hline & & 1978 & $87 \%$ & Rural & \\
\hline & & 1983 & $80 \%$ & & $\begin{array}{l}\text { Norin, Carlsson, \& Korner } \\
\text { (1984) }\end{array}$ \\
\hline \multirow[t]{10}{*}{ Switzerland } & $1 / 76$ & prelaw & $35 \%$ & & Fisher (1980) \\
\hline & & $2 / 76$ & $95 \%$ & Expressway drivers & \\
\hline & & $2 / 76$ & $92 \%$ & Rural drivers & \\
\hline & & $2 / 76$ & $89 \%$ & Urban drivers & \\
\hline & & $9 / 78$ & $64 \%$ & Expressway drtvers & \\
\hline & & $9 / 78$ & $46 \%$ & Rural drivers & \\
\hline & & $9 / 78$ & $33 \%$ & Urban drivers & \\
\hline & $7 / 81^{b}$ & 1982 & $77 \%$ & Expressways & And reasson (1983) \\
\hline & & 1982 & $76 \%$ & Rural & \\
\hline & & 1982 & $62 \%$ & Urban & \\
\hline \multirow[t]{8}{*}{ West Germany } & $1 / 76$ & $8 / 75$ & $28 \%$ & & Federal Institute of \\
\hline & & $11 / 75$ & $32 \%$ & & Streets (cited in \\
\hline & & $1 / 76$ & $50 \%$ & & Fisher, 1980) \\
\hline & & $3 / 77$ & $46 \%$ & & \\
\hline & & $9 / 77$ & $48 \%$ & & \\
\hline & & $9 / 78$ & $58 \%$ & & \\
\hline & & $9 / 84$ & $92 \%$ & Fines began $8 / 85$ & Marburger (1986) \\
\hline & & $3 / 86$ & $94 \%$ & & \\
\hline
\end{tabular}

aprivers and front-seat passengers unless otherwise noted. 'Switzerland's 1976 law declared inval id by the Supreme court in $9 / 77$ and reinstated by the government on $7 / 1 / 81$.

analyses. State-specific VMT figures by month were obtained from the U.S. Federal Highway Administration and are based on traffic counter and motor fuel sales data. Pre- and postlaw means and standard deviations for each major time-series analyzed are shown in Table 5 .

\section{Statistical Methods}

We used the time-series intervention analysis methods of Box and Tiao (1975) and Box and Jenkins (1976), On a conceptual level, the analytic strategy involves explaining as much as possible of the variance in fatality 
TABLE 3

EFFECTS OF NON-U.S. SEAT BELT LAWS ON FATALITIES

\begin{tabular}{|c|c|c|c|c|}
\hline Jurisdiction & $\begin{array}{l}\text { Effective } \\
\text { Month }\end{array}$ & $\begin{array}{l}\text { Post-1aw } \\
\text { Months }\end{array}$ & $\begin{array}{c}\text { Fatality } \\
\text { Change }\end{array}$ & Investigators \\
\hline \multicolumn{5}{|l|}{ Australia } \\
\hline \multirow[t]{6}{*}{ Victoria } & $1 / 71$ & 9 & $-15 * * *$ & Foldvary \&ane (1974) \\
\hline & & 48 & $-37 \%$ & Trinca Dooley $(1977)$ \\
\hline & & 10 & $-15 \%$ & Andreassend (1976) \\
\hline & & 12 & $-15 \%$ & Joubert (1979) \\
\hline & & 84 & $* * *$ & McDemott \& Hough (1979) \\
\hline & & 144 & $-60 \%$ & Trinca (1984) \\
\hline \multirow[t]{2}{*}{ Queenst and } & $1 / 72$ & & $-14 \%$ & Johinke (1977) \\
\hline & & & $-46 x$ & Bhattacharyya Layton (1979) \\
\hline South Australfa & $11 / 71$ & 12 & $-8 \%$ & Crinion et al. (1975) \\
\hline Australia (overall) & & & $-20 \%$ & Fisher (1980) \\
\hline \multicolumn{5}{|l|}{ Canada } \\
\hline \multirow[t]{3}{*}{ Ontario } & $1 / 76$ & 6 & $-13 x$ & Snow (1979) \\
\hline & & 72 & $-26 \%$ & Jonah \& Lawson (1984) \\
\hline & & 72 & $-12 \%$ & Hedl und (1986) \\
\hline \multirow[t]{4}{*}{ Sa skatchewan } & $7 / 77$ & 12 & $-20 \%$ & Shiels (1978) \\
\hline & & 52 & $-37 \%$ & Jonah L Lawson $(1984)^{\mathrm{a}}$ \\
\hline & & & $-35 *^{* *}$ & \\
\hline & & 52 & -298 & Hedl und (1986) \\
\hline \multirow[t]{2}{*}{ British Columbia } & $10 / 77$ & 51 & $-30 \% *$ & Jonah Lawson (1984) \\
\hline & & 60 & $-52 x$ & Hedl und (1986) \\
\hline \multirow[t]{2}{*}{ Quebec } & $8 / 76$ & 65 & $-17 \%$ & Jonah \& Lawson (1984) \\
\hline & & 65 & $-18 \%$ & Hed und $(1986)$ \\
\hline \multirow[t]{2}{*}{ Denmark } & $1 / 76$ & 12 & -17 & Nordic Traffic Safety Council (1984) \\
\hline & & 12 & $-13 \%$ & Hedl und $(1986)$ \\
\hline \multirow[t]{3}{*}{ England } & $2 / 83$ & 11 & $-25 \%$ & Mackay (1984b) \\
\hline & & 3 & $-80 \% * \star \star \star *$ & Pye Waters (1984) \\
\hline & & 23 & $\begin{array}{l}-18 x^{b} \\
-25 x^{c}\end{array}$ & Durbin \& Harvey (1985) \\
\hline
\end{tabular}




\begin{tabular}{|c|c|c|c|c|}
\hline Jurisdiction & $\begin{array}{l}\text { Effective } \\
\text { Nonth }\end{array}$ & $\begin{array}{c}\text { Post-1aw } \\
\text { Months }\end{array}$ & $\begin{array}{c}\text { Fatality } \\
\text { Change }\end{array}$ & Investigators \\
\hline \multirow[t]{2}{*}{ France } & $7 / 73$ & 23 & -218 & Chodkiewicz \& Dubarry (1977) \\
\hline & & 114 & $-50 \%$ & Hartemann et al. (1984) \\
\hline Irel and & $2 / 79$ & 11 & $-0.7 \%$ & Hearne (1981) \\
\hline \multirow[t]{2}{*}{ Israel } & $7 / 75$ & 30 & $\begin{array}{l}-42 \%^{b} \\
-44 \%^{c}\end{array}$ & Hakkert et al. (1981) \\
\hline & & 30 & $-41 \%$ & Hedl und (1986) \\
\hline \multirow[t]{2}{*}{ New Zealand } & $6 / 72$ & 24 & $+3 x^{d}$ & Toomath (1977) \\
\hline & & 24 & $-43 \%$ & Hed? und (1986) \\
\hline \multirow[t]{3}{*}{ Norway } & $9 / 79$ & & $-21 \%$ & $\begin{array}{l}\text { McCarthy, Tayl or, Sandford, } \\
\text { \& Lange (1984) }\end{array}$ \\
\hline & & 12 & $-10 \%$ & Nordic Traffic Safety Council (1984) \\
\hline & $9 / 75$ & 12 & $-29 \%$ & Hedl und (1986) \\
\hline South Africa & $12 / 77$ & & $-8 \%$ & McCarthy et al . (1984) \\
\hline \multirow[t]{3}{*}{ Sweden } & $1 / 75$ & & $-14 \%$ & Mccarthy et al. (1984) \\
\hline & & 12 & $-12 \%$ & Boht in (1973) \\
\hline & & 12 & $-12 \%$ & Norin et al. (1984) \\
\hline \multirow[t]{2}{*}{ Sunitzerl and } & $1 / 76$ & 12 & $-12 \%$ & Fisher (1980) \\
\hline & $7 / 81^{e}$ & 12 & $-15 \%$ & Hedl und (1986) \\
\hline West Gemany & $1 / 76$ & $6^{f}$ & $-25 \%$ & hedl und (1986) \\
\hline
\end{tabular}

\footnotetext{
a Crash data for the perfod $7 / 1 / 77$ to $12 / 31 / 82$ were analyzed; actual fatality rates were significantly different from predicted rates only in $1980(\mathrm{p}<.10)$ and 1981 ( $<<.05)$ Grivers only. Front-seat passengers. In contrast, nonoccupant fatalities increased almost $40 \%$ during this period. "Switzerland's 1976 law declared inval id by the Supreme Court in $9 / 77$ and reinstated by the government on $7 / 1 / 81$. This study compared the pre- and postfine period $1-6 / 84$ to $1-6 / 85$.

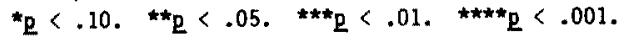

rates on the basis of the past history of those rates, before attributing any of the variance to an exogenous variable, such as implementation of a seat belt law. This approach of intervention analysis was particularly appropriate for the current study, because the objective was to identify significant shifts in fatality rates associated with seat belt laws, independent of observed regularities in the history of each series. Ordinary leastsquares regression and other commonly used statistical procedures were not appropriate for the present study because they assume independent observations. However, a series of observations over time, such as the fatality rate series analyzed here, are highly auto- 
TABLE 4

EFFECTS OF U.S. SEAT BELT LAWS ON FATALITITES

\begin{tabular}{|c|c|c|c|c|}
\hline Jurf sdiction & $\begin{array}{c}\text { Effoctive } \\
\text { Wonth }\end{array}$ & $\begin{array}{c}\text { Post-1uw } \\
\text { Months }\end{array}$ & $\begin{array}{c}\text { Fatallty } \\
\text { Change }\end{array}$ & Investi gators \\
\hline \multirow[t]{7}{*}{ New York } & $12 / 84$ & 9 & $-98 * *$ & Lund, Pollner, \&illiams (1986) \\
\hline & & 9 & $-15 x^{+}$ & Hedl und (1986) \\
\hline & & 6 & $-20 x^{+}$ & Latimer Lave (1987) \\
\hline & & 3 & -278 & Pace et al. (1986) \\
\hline & & 13 & $-5 \% * * *$ & Lund, Zador, \& Pollner (1986) \\
\hline & & 13 & $-8 * *$ & Campbell et al. (1986) \\
\hline & & 19 & $-7 x$ & Hoxie Skinner (2987) \\
\hline \multirow[t]{3}{*}{ New Jersey } & $3 / 85$ & 10 & $-4 \%$ & Lund, Zador, Poliner (1986) \\
\hline & & 10 & $-6 \%$ & Campbell et al. (1986) \\
\hline & & 16 & $-2 x$ & Hoxte \& Skinner (1987) \\
\hline \multirow[t]{4}{*}{ Michi gan } & $7 / 85$ & 12 & $-10 x$ & Hagenaar, Maybee, Sullivan (1987) \\
\hline & & 6 & $-4 *$ & Lund, Zador, \& Pollner (1986) \\
\hline & & 6 & $-16 * *$ & Campbell et a1. (1986) \\
\hline & & 12 & $-14 \% *$ & Hoxie \& Skinner (1987) \\
\hline \multirow[t]{4}{*}{ Illinois } & $7 / 85$ & 9 & $-3 x^{+}$ & Mortimer (1986) \\
\hline & & 6 & $-7 \%$ & Lund, Zador, \& Polliner (1986) \\
\hline & $7 / 86$ & 6 & $-9 \%$ & Campbell et al. (1986) \\
\hline & & 12 & $-1 \%$ & Hoxie Skinner (1987) \\
\hline \multirow[t]{2}{*}{ Texas } & $9 / 85$ & 4 & $-18 \% * * *$ & Campbell et al. (1986) \\
\hline & & 10 & $-18 \psi^{* \star * \star}$ & Hoxie Skinner (1987) \\
\hline Nebraska & $9 / 85$ & 4 & $-11 \%$ & Campbell et al, (1986) \\
\hline \multirow[t]{2}{*}{ Missourt } & $9 / 85$ & 3 & $+5 \%$ & Campbell et a1. (1986) \\
\hline & & 9 & $+18 \% \star \star \star$ & Hoxie Skinner (1987) \\
\hline \multirow[t]{2}{*}{ North Carolina } & $10 / 85$ & 3 & $-0.4 \%$ & Canpbe1l et al. (1986) \\
\hline & & 9 & $-5 \%$ & Hoxie \& Skinner (1987) \\
\hline \multirow[t]{3}{*}{$A 11^{a}$} & $b$ & $b$ & $-7 \%^{+}$ & Partyka (1987) \\
\hline & & & $-10 \%$ & Campbell et al. (1986) \\
\hline & & & $-6 x^{c}$ & Hoxle Skinner $(1987)$ \\
\hline
\end{tabular}

${ }^{a}$ NY, NJ, IL, MI, TX, MB, MO, NC. 'Different for each state, depending on date law enacted. "Total excludes NB.

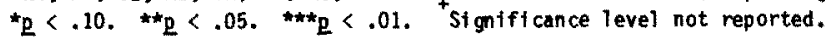




\begin{tabular}{|c|c|c|c|c|}
\hline \multirow[b]{2}{*}{ Comparison } & \multicolumn{2}{|c|}{ Prelaw } & \multicolumn{2}{|c|}{ Postlaw } \\
\hline & Mean & $\underline{S D}$ & Mean & $\underline{S D}$ \\
\hline \multicolumn{5}{|l|}{ Rate of front-seat fatalities per MI } \\
\hline New York & 158.14 & 32.79 & 113.87 & 22.39 \\
\hline Hew Jersey & 116.63 & 21.30 & 99.63 & 14.40 \\
\hline Michigan & 165.84 & 36.06 & 132.76 & 26.90 \\
\hline Illinois & 168.42 & 38.61 & 123.25 & 17.07 \\
\hline Texas & 213.45 & 34.39 & 139.70 & 9.01 \\
\hline Nebraska & 182.26 & 61.46 & 155.61 & 38.09 \\
\hline Missourt & 201.77 & 45.75 & 189.31 & 26.08 \\
\hline North Carolina & 211.44 & 37.45 & 201.84 & 22.76 \\
\hline \multicolumn{5}{|l|}{ Relative rate of front-seat fatalities per $\mathrm{MT}$} \\
\hline New York vs. Pennsylvania & .95 & .14 & .75 & .11 \\
\hline New Jersey vs. Maryland & .89 & .24 & .78 & .19 \\
\hline Michigan vs. ohio & 1.07 & .21 & .97 & .19 \\
\hline lllinofs vs. Indiana & 1.10 & .35 & .84 & .14 \\
\hline Texas vs. Georgia & 1.14 & .23 & .88 & .18 \\
\hline Nebraska vs. Kansas & 1.01 & .44 & .84 & .24 \\
\hline Missour vs. Tennessee & 1.17 & .32 & .89 & .21 \\
\hline North Carolina vs. Virginia & 1.40 & .33 & 1.46 & .22 \\
\hline \multicolumn{5}{|c|}{ Aggregate relative rate of front-seat fatalities per VMT } \\
\hline Eight belt-1aw vs. elght comparison states & 1.07 & .09 & .89 & .04 \\
\hline \multicolumn{5}{|l|}{ Aggregate rate of rear-seat fatalities per yMI } \\
\hline Eight belt law states & 12.48 & 3.18 & 9.54 & 1.24 \\
\hline \multicolumn{5}{|l|}{ Agaregate rate of nonoccupant fatalities per WMT } \\
\hline Eight belt law states & 66.92 & 18.21 & 41.90 & 3.92 \\
\hline \multicolumn{5}{|l|}{ Aggregate rate of front-seat fatalittes per WMT by } \\
\hline \multicolumn{5}{|l|}{ Enforcement provision } \\
\hline Primary enforcement & 191.14 & 29.48 & 128.76 & 8.86 \\
\hline Secondary enforcement & 153.59 & 25.39 & 121.19 & 13.98 \\
\hline
\end{tabular}

correlated, violating the assumption of independence and leading to biased standard error estimates using conventional methods.

Baseline Auto-regressive Integrated Moving Average (ARIMA) models were iteratively developed for each time series, repeatedly going through cycles of specifying a model, estimating it, and evaluating its adequacy in terms of accounting for all significant autocorrelation patterns in the series. All of the time series were natural-logarithm transformed prior to parameter estimation to reduce heteroscedasticity. All of the final models met the multiple criteria for model adequacy identified by Box and Jenkins (1976), including significant noise model parameters, low correlations among parameters, and insignificant residual autocorrelations.

Transfer functions were added to the noise models to test for effects of seat belt laws. Given the short postlaw period for which data were available, simple shift transfer function models were used to represent potential effects of the belt laws. Additional transfer functions were added to the 
FIGURE 1

\section{PERCENT CHANGE IN RATE OF FATALITIES PER VMT \\ ASSOCIATED WITH SEAT BELT LAWS: \\ FRONT-SEAT OCCUPANTS AGE 10 AND OVER}

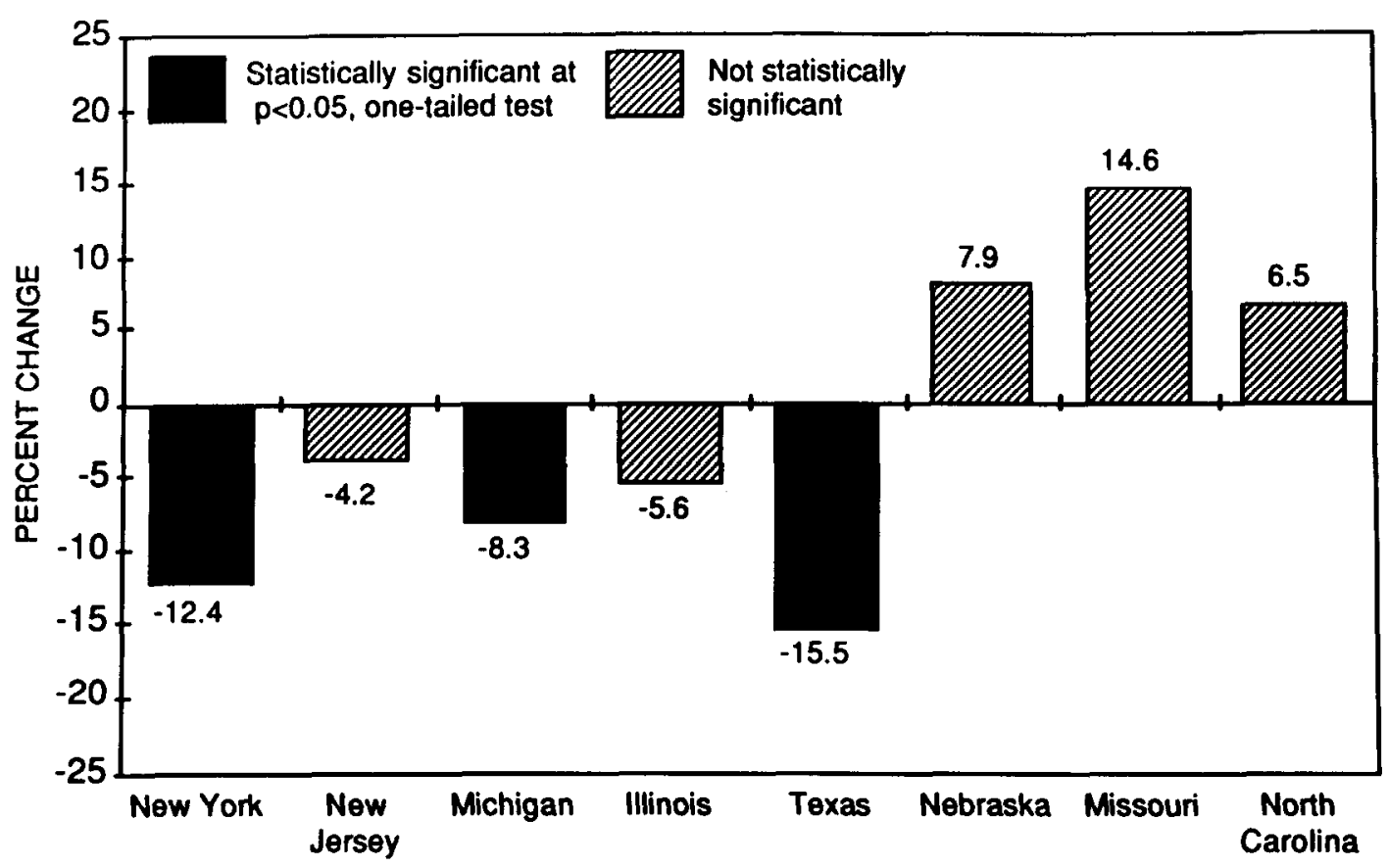

models for selected time series. The substantial decline in the fatality rate in 1982 in most of the states was controlled by including a simple shift transfer function. The 1982 decline was due to a variety of factors, including a major economic recession, campaigns to reduce alcohol-impaired driving, and changes in the age structure of the population (Hedlund, Arnold, Cerelli, Partyka, \& Hoxie, 1984). Our ubjective was not to fully elucidate the causal structure underlying those fatality reductions, but rather to statistically control for those reductions when estimating the effects of recent compulsory seat belt laws.

Because the models are intrinsically nonlinear, the Gauss-Marquardt backcasting algorithm implemented in the software package BMDP2T was used to estimate the parameters (Dixon et al., 1983). All parameter estimates in the logarithm metric were converted to an estimated percent change in the series after the seat belt law, from levels expected given baseline patterns, using $\left(e^{\omega}-1\right) 100$. Final statistical models for aggregate series are shown in the Appendix.

\section{RESULTS}

Significant declines in the rate of frontseat occupant deaths per VMT occurred in three of the eight states with mandatory seat belt laws (Figure 1). The fatality rate declined $8.3 \%$ in Michigan, $12.4 \%$ in New York, and $15.5 \%$ in Texas. Intervention parameter estimates were in the expected direction (though not significant) in New Jersey and Illinois. The fatality rate increased in Missouri, Nebraska, and North Carolina, but only in Missouri was the estimated increase larger than two standard errors. ${ }^{2}$ While these analyses control for long-term trends and cycles within each state, and control for changes in exposure via rates per

2Technically not statistically significant because we hypothesized a fatality reduction following implementation of belt laws and thus used one-tailed tests. 
VMT, these state-specific changes in fatalities may still simply reflect broader regional or national changes due to other factors. To ensure that observed fatality changes were associated with the seat belt laws and not other factors, we analyzed the rate of fatalities per VMT in a state with a new belt law relative to the rate of fatalities per VMT in a neighboring state without a belt law during the period studied. In other words, the fatality rate in the target state was divided by the rate in the comparison state.

Analyses of the relative rates again indicated significant declines in fatalities associated with seat belt laws in three of the eight states: New York, 7.1\%; New Jersey, $24.5 \%$; and Nebraska, $19.3 \%$ (Figure 2). However, as noted in the previous paragraph, two of these three, (New Jersey and Nebraska) showed no significant decrease when examining the state alone, without taking into account the experience in comparison states. In addition to significant reductions in the relative rates of fatalities in three states, time-series modeling produced estimates in the expected direction (although not significant) in an additional four states.

Clearly, the small number of postlaw data points available (9 to 19 months), and the substantial baseline variability in fatality rates over time, results in moderately large standard errors and what appear to be inconsistent results across states. To reduce this background variation, we combined the eight belt-law states, and estimated the aggregate effect of the belt laws in these eight states. The state-specific time series were aligned on the month each state's belt law took effect, and the number of fatalities and amount of vehicle mileage traveled were summed. The eight comparison states were similarly summed. The result was a time series in which each month no longer represented a specific month in time, but rather represented the ordinal month from the point at which belt laws were implemented. Dividing the fatality rate per VMT for the belt-law states as a group by the fatality rate per VMT for the comparison states as a

FIGURE 2

PERCENT CHANGE IN RATE OF FATALITIES PER VMT

AMONG FRONT-SEAT OCCUPANTS AGE 10 AND OVER:

BELT-LAW STATES RELATIVE TO COMPARISON STATES

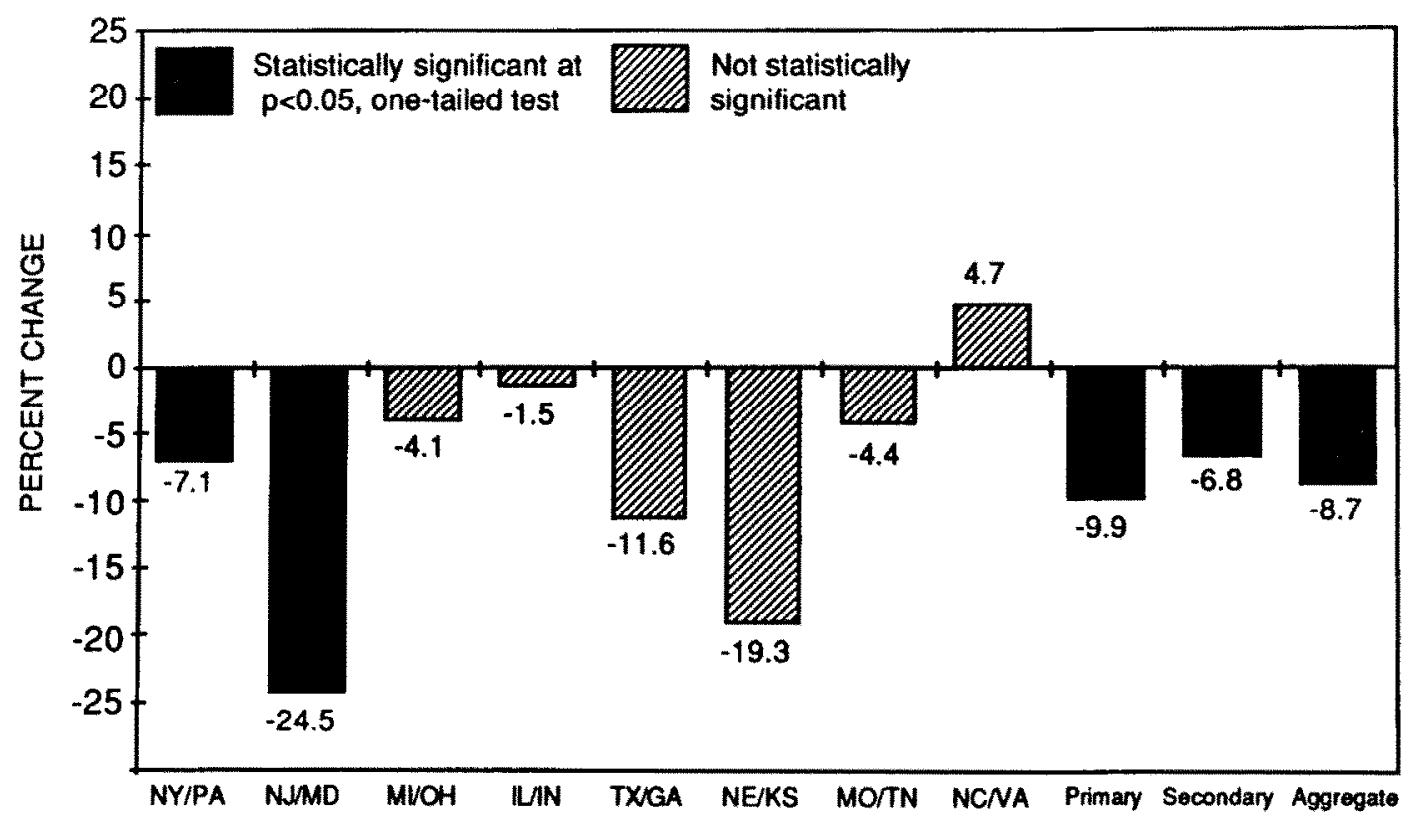


group resulted in an aggregate relative rate (Figure 3). Time-series modeling of the aggregate relative rate estimated a statistically significant $8.7 \%$ decline associated with belt laws in these states.

One obvious explanation for the differential effects of seat belt laws across states is the size of the change in belt use caused by the law. Most states experienced an increase in belt use from about $16 \%$ before to $45 \%$ a few months after the laws took effect (Table 1). Texas had a larger than average increase in belt use (from $15 \%$ to $66 \%$ ) and Illinois a smaller than average increase (from $16 \%$ to $30 \%$ ). Given the different survey methods used in each state, and the standard errors of our estimates of belt law effects on fatalities, we are not in a position to argue that cross-state differences in our fatality reduction estimates reflect differences in belt use rates across states.

Nevertheless, specific provisions of the law, such as primary versus secondary enforcement, ${ }^{3}$ and the intensity with which it is enforced are expected to influence belt use rates. To take into account these major differences in the laws across states, we conducted time-series analyses of two groups of states-states with primary enforcement versus states with secondary enforcement

3Secondary enforcement means that police officers may only issue citations for failure to use belts if the motorist is first stopped for some other offense. That is, a motorist may not be stopped solely for failure to use seat belts.

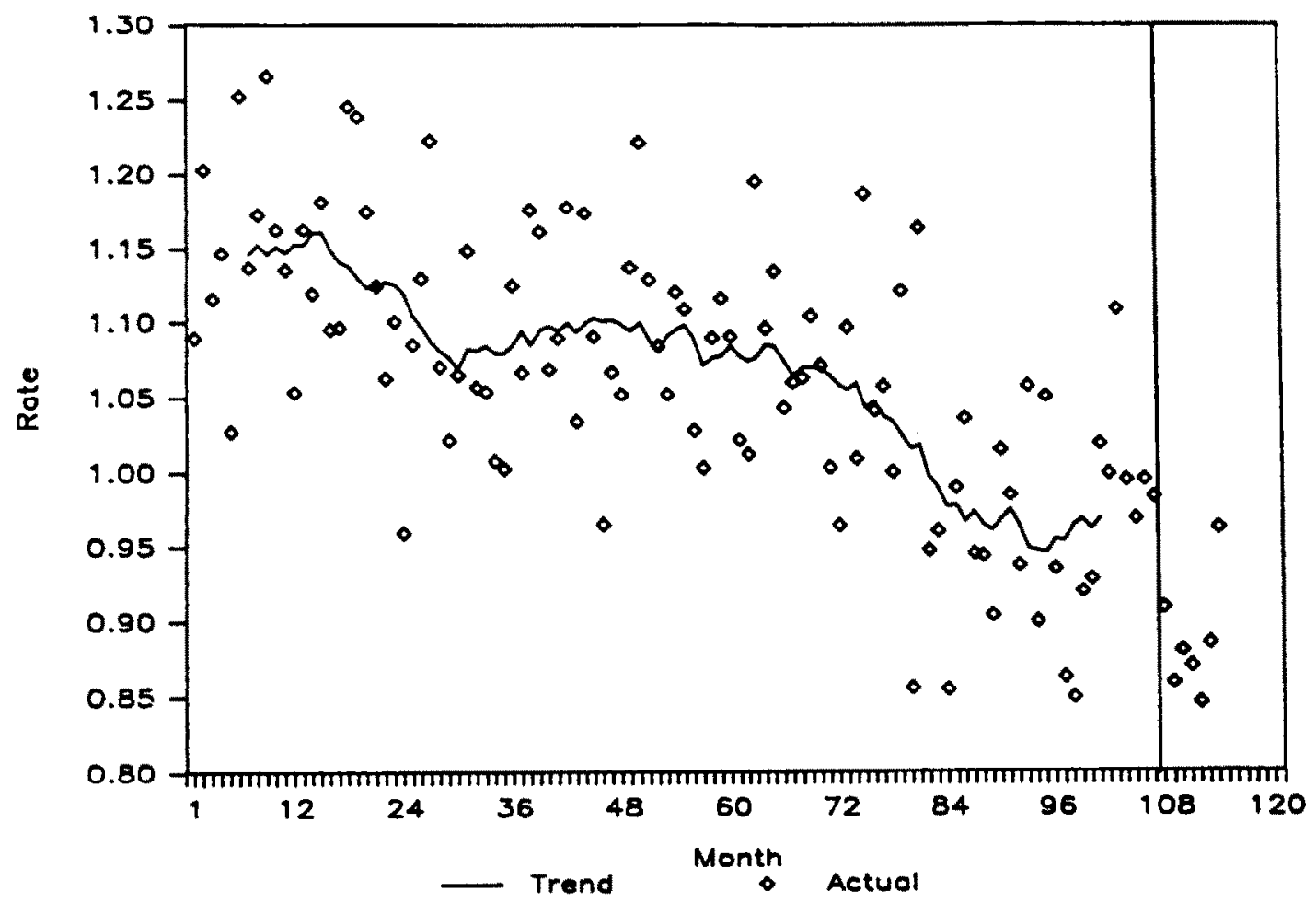

${ }^{\mathrm{a}}$ Time series for each state were aligned on the month in which seat belt use became compulsory (month 108). 
only. Because North Carolina and Missouri were not actively enforcing their laws during this period, they were excluded from these analyses. Results indicated a significant $9.9 \%$ fatality reduction in the primary enforcement states and a significant $6.7 \%$ fatality reduction in secondary enforcement states (Figure 2). As expected, states with primary enforcement experienced larger fatality reductions than states limited to secondary enforcement. However, it is worth noting that clear benefits also accrued from secondary enforcement belt laws, provided citations were actually issued to violators.

Finally, in addition to controlling for other plausible explanations for observed fatality declines by including comparison states, we conducted time-serics analyses of rates of rear-seat occupant deaths and nonoccupant (motorcycle, pedalcycle, and pedestrian) deaths in the states with seat belt laws. All of the laws examined here are limited to front-seat occupants; as a result, rear-seat occupants along with nonoccupants serve as useful comparison groups. Analyses of aggregate fatality rates for the eight belt-law states revealed no change in fatality rates among either rear-seat occupants or nonoccupants. This result substantially increased our level of confidence in attributing observed declines in front-seat fatalities to the seat belt laws.

\section{DISCUSSION}

Our results confirm that laws requiring seat belt use can significantly reduce rates of motor vehicle fatalities. However, one cannot expect the fatality declines to be clearly demonstrable within single jurisdictions a short time after the laws are implemented. The nature of fatality trends over time, and the amount of unpredictable variation in number of deaths from month to month, means that a minimum of a $6 \%$ to $10 \%$ reduction over a 6 - to 12 -month period is required before the reduction can be reliably identified. Despite the lack of statistical significance for the estimated effects of seat belt laws in some jurisdictions, it is premature to conclude that laws in those states had no effect. As additional data become available, increasing the statistical power of analytic techniques used, some of the statespecific estimates obtained in the current study may become statistically significant. Results from our most powerful analyses, those involving aggregate effects across several states, clearly demonstrate significant fatality reductions. Moreover, use of comparison states and comparison groups not directly affected by the seat belt laws increases our confidence in interpreting the observed declines as caused by the mandatory seat belt use laws.

In terms of the magnitude of the effects of compulsory belt use laws, one can expect a U.S. law that permits primary enforcement and is actually enforced at moderate levels to result in about a $10 \%$ reduction in traffic fatalities. A law that permits secondary enforcement only or is enforced at very low levels will have less effect. Although some advocates of compulsory seat belt use have indicated that substantially larger declines in traffic fatalities would result, a $10 \%$ decline in a leading cause of death for the entire population represents a resounding public policy success. How many major programs aimed at reducing disease and injury can document an immediate $10 \%$ decline in mortality due to that cause of death across an entire population of millions of people? Moreover, effective implementation of a compulsory seat belt use policy requires minimal expenditure of resources when compared to efforts to reduce mortality attributable to other leading causes of death (e.g., cardiovascular disease, cancer).

Despite the clear success of compulsory seat belt laws to date, much more remains to be done. As noted earlier, belt use in the U.S. typically peaks within a month or two of implementation of belt laws, partially decaying after that point. Special enforcement efforts not only can arrest that decline, but further increase belt use rates, at least temporarily (Williams, Preusser, Blumberg, \& Lund, in press). Clearly, our results demonstrate that belt laws with primary rather than secondary enforcement provisions are needed. We believe that rigorous enforcement of a primary seat belt law in the U.S. can achicve and maintain belt use rates of approximately $60 \%$, in contrast to less than $20 \%$ under the most favorable conditions 
without compulsory use (i.e., extensive education and public information programs).

Even if asymptotic belt use of $60 \%$ were achieved throughout the U.S., declines in traffic fatalities of more than $20 \%$ are extremely unlikely. This is because of the differential between belt users and nonusers; that is, those at highest risk of involvement in serious traffic crashes are least likely to use belts (Evans \& Wasielewski, 1983). Therefore, other avenues of reducing traffic crash-induced injury and death that do not require action on the part of each individual driver (such as airbags) must be pursued simultaneously with efforts to implement and enforce mandatory belt use laws.

\section{REFERENCES}

Andreassend, D. C. (1976). Victoria and the seat belt law, 1971. Human Factors, 18, 593-600.

Andreasson, R. (1983). Switzerland shows what a seat belt law can mean. Journal of Traffic Medicine, 11, 25-26.

Arora, H. R. (1982). Seat belt use by Canadian drivers, November 1981. Ottawa: Road Safety Directorate, Transport Canada.

Arora, H. R. (1985). Seat belt use by Canadian drivers, November 1983. Ottawa: Road Safety Directorate, Transport Canada.

Ashton, S. J., Mackay, G. M., \& Camm, S. (1983). Seat belt use in Britain under voluntary and mandatory conditions. In 27th Annual Proceedings of the American Association for Automotive Medicine (pp. 6575). Morton Grove, IL: American Association for Automotive Medicine.

B. C. Rescarch. (1983). Restraint usage in British Columbia during 80\% clicks, June 3 and 4, 1983: Executive summary. Vancouver: Insurance Corporation of British Columbia.

Bergen, A. T., Watson, L. G., Rivett, D. E., \& Shiels, A. C. (1979). The effect on injury and fatality rates due to seat belt usage in Saskatchewan. In $23 \mathrm{rd} \mathrm{An-}$ nual Proceedings of the American Association for Automotive Medicine (pp. 412-425). Morton Grove, IL: American Association for Automotive Medicine.

Bhattacharyya, M. N., \& Layton, A. P. (1979). Effects of seat belt legislation on the Queensland road toll An Australian case study in intervention analysis. Journal of the American Statistical Association. 74, 596-603.

Bohlin, N. I. (1973). Twenty years of safety belt experience and the effect of safety belt legislation in Sweden. In 79 International Symposium on Seat Belts in Tokyo (pp. 116-125). Tokyo: Japanese Council of Traffic Science.

Box, G. E. P., \& Jenkins, G. M. (1976). Time series analysis: Forecasting and Control (rev. ed.). San Francisco: Holden-Day.

Box, G. E. P., \& Tiao, G. C. (1975). Intervention anal- ysis with applications to economic and environmental problems. Journal of the American Statistical Association, 70, 70-79.

Bunch, N. G., Hatfield, N. J., Hinshaw, W. M., \& Womack, K. N. (1986). Observed front seat occupant restraint use in fourteen Texas cities before and after safety belt use legislation. College Station: Texas Transportation Institute, Texas A\&M University System.

Campbell, B. J., Stewart, J. R., \& Campbell, F. A. (1986). Early results of seat belt legislation in the United States of America. Chapel Hill: The University of North Carolina Highway Safety Research Center.

Chodkiewicz, J. P., \& Dubarry, D. (1977). Effects of mandatory seat belt legislation in France. In Proceedings of the Sixth International Conference of the International Association for Accident and Traffic Medicine (pp. 40-44). Stockholm: International Association for Accident and Traffic Medicine.

Cook, T. D., \& Campbell, D. T. (1979). Quasi-experimentation: Design and analysis issues for field settings. Chicago: Rand-McNally.

Crinion, J. D., Foldvary, L. A., \& Lane, J. C. (1975). The effect on casualties of a compulsory seat belt wearing law in South Australia. Accident Analysis and Prevention, 7, 81-89.

DataCom Opinion Research Corp. (1984). Manitoba shoulder belt observation survey, June, 1984: Summary of results. Winnipeg: Manitoba Traffic Safety Committee.

Dixon, W. J., Brown, M. B., Engelman, L., Frane, J. W., Hill, M. A., Jennrick, R. I., \& Toporek, J. D. (1983). BMDP statistical software: 1983 printing with additions. Berkeley: University of California Press.

Durbin, J., \& Harvey, A. C. (1985). The effects of seat belt legislation on road casualties in Great Britain: Report on assessment of the statistical evidence. Compulsory seat belt wearing report by the Department of Transport, Annex (pp. 1-A46). London: Her Majesty's Stationery Office.

Evans, L. (1986). The effectiveness of safety belts in preventing fatalities. Accident Analysis and Prevention, 18, 229-241.

Evans, L., \& Wasielewski, P. (1983). Risky driving related to driver and vehicle characteristics. Accident Analysis and Prevention, 15, 121-136.

Fernie, B. E. (1980). Compulsory wearing of seat belts in South Africa: Interim evaluation of effectiveness. Pretoria: National Institute for Transport and Road Research.

Fisher, F. G. (1980). Effectiveness of safety belt usage laws (PB80 209901). Washington, DC: U.S. Department of Transportation, NHTSA.

Foldvary, L. A., \& Lane, J. C. (1974). The effectiveness of compulsory wearing of seat belts in casualty reduction. Accident Analysis and Prevention, 6, 5981 .

Gerondeau, C. (1979). Enaction of seat belt use laws in France. In '79 International Symposium on Seat Belts in Tokyo (pp. 189-193). Tokyo: Council on Traffic Science.

Gerondeau, C. (1981, June). Mandatory seat belt usage: The French Experience 1973/1981. Paper pre- 
sented at the International Symposium on Occupant Restraints, Toronto.

Hakkert, A. S., Zaidel, D. M., \& Sarelle, E. (1981). Patterns of safety belt usage following introduction of a safety belt wearing law. Accident Analysis and Prevention, 13, 65-82.

Hartemann, F., Henry, C., Faverjon, G., Tarriere, C., Got, C., \& Patel, A. (1984). Ten years of safety due to the three-point belt. In Advances in restraint systems: Design, performance and usage (pp. 7-13). Warrendale, PA: Society of Automotive Engineers.

Hatfield, N. J., Hinshaw, W. M., Bunch, N. G., \& Bremmer, R. (1985). Observed pre-law safety belt use by adult front seat occupants in twelve Texas cities. College Station: Texas Transportation Institute, Texas A\&M University System.

Hearne, R. (1981). The initial impact of the safety belt legislation in Ireland. Dublin: An Foras Forbartha, The National Institute for Physical Planning and Construction Research.

Hedlund, J. (1986). Casualty reductions: Results from safety belt use laws. In Effectiveness of safety bell use laws: A multinational examination (pp. 73-97). Washington, DC: NHTSA. (NTIS No. DOT HS 807018)

Hedlund, J., Arnold, R., Cerrelli, E., Partyka, S., \& Hoxie, P. (1984). An assessment of the 1982 traffic fatality decrease. Accident Analysis and Prevention, $16,247-261$.

Hoxie, P., \& Skinner, B. (1987). Fatality reductions from mandatory seatbelt usage laws. In Restraint Technologies: Front seat occupant protection (pp. 199-203). Warrendale, PA: Society of Automotive Engineers.

Insurance Institute for Highway Safety (IIHS). (1987a). North Carolina leads nation in seat belt use. Status Report, 22(3), 5.

IIHS. (1987b). Seat belt use rates continue to vary among the states. Status Report, 22(3), 6 .

Johinke, A. K. (1977). South Australian experience with the compulsory wearing of seat belts. In Proceedings of the Sixth International Conference of the International Association for Accident and Traffic Medicine (pp. 125-129). Stockholm: International Association for Accident and Traffic Medicine.

Jonah, B. A., \& Lawson, J. J. (1984). The effectiveness of Canadian mandatory seat belt laws. Accident Analysis and Prevention, 16, 433-450.

Jonah, B. A., \& Lawson, J. J. (1986). Safety belt use rates and user characteristics. In Effectiveness of safety belt use laws: A multinational examination (pp. 43-72). Washington, DC: NHTSA. (NTIS No. DOT HS 807018)

Joubert, P. N. (1979). Development and effects of seat belt laws in Australia. In '79 International Symposium on Seat Belts in Tokyo (pp. 126-131). Tokyo: Japanese Council of Traffic Science.

Latimer, E. A., \& Lave, L. B. (1987). Initial effects of the New York State auto safety belt law. American Journal of Public Health, 77, 183-186.

Lund, A. K., Pollner, J., \& Williams, A. F. (1986). Preliminary effects of mandatory seat belt use laws: Science versus politics. Washington, DC: IIHS.

Lund, A. K., Zador, P., \& Pollner, J. (1986). Motor vehicle occupant fatalities in four states with seat belt use laws. Washington, DC: IIHS.

Mackay, M. (1984a). Legislation for seat belt use in Britain. In Advances in belt restraint systems: Design, performance, and usage (pp. 153-160). Warrendale, PA: Society of Automotive Engineers.

Mackay, M. (1984b). Seat belt use under voluntary and mandatory conditions and its effect on casualties. In Symposium on human behavior and traffic safety (pp. 259-283). Warren, MI: General Motors Research Laboratories.

Manders, S. L. (1984). Restraint use in Victoria: Resulls of March 1984 survey. Melbourne, Australia: Road Traffic Authority.

Marburger, E. A. (1986). Safety belt use rates. In Effectiveness of safety belt use laws: A multinational examination (pp. 25-41). Washington, DC: NHTSA. (NTIS No. DOT HS 80701)

Matthews, M. L. (1982). Seat belt usage in Ontario four years after mandatory legislation. Accident Analysis and Prevention, 14, 431-438.

McCarthy, R. L., Taylor, R. K., Sanford, S. B., \& Lange, R. C. (1984), Seat belts: Effectiventess of mandatory use requirements. In Advances in belt restraint systems: Design, performance, and usage (pp. 161-171). Warrendale, PA: Society of Automotive Engineers.

McDermott, F. T., \& Hough, D. F. (1979). Reduction in road fatalities and injuries after legislation for compulsory wearing of seat belts: Experience in Victoria and the rest of Australia. British Journal of Surgery, 66, 518-521.

Mortimer, R, G. (1986). Seat belt use by fronl seat occupants in Illinois. Champaign: University of Illinois at Urbana-Champaign, Department of Health and Safety Studies.

Murray, E. (1984). Results of seat belt survey, July 1984 (research report 27). St. Johns, Newfoundland: Highway Planning and Research, Department of Transportation.

New Brunswick Department of Transportation. (1984). Results of seat belt survey, August 1984. Fredericton, New Brunswick: Motor Vehicle Administration and Public Safety Coordination.

Nordic Traffic Safety Council. (1984). Restraints for adults and children in the back seats of private cars (appendix II and III, report 37A). Oslo: Author.

Norin, H., Carlsson, G., \& Korner, J. (1984). Seat belt usage in Sweden and its injury reducing effect. In Advances in belt restraint systems: Design, performance, and usage (pp. 15-28). Warrendale, PA: Society of Automotive Engineers.

O'Day, J., \& Flora, J. (1982). Alternative measures of restraint system effectiveness: Interaction with crash severity figures (SAE paper 820798). Troy, MI: Society of Automotive Engineers.

Oranen, L. (1977). Use of safety belts: Investigation into effect of compulsory use of safety belts on volume of use. Helsinki: Central Organization for Traffic Safety, Research Department.

Oranen, L., \& Koivurova, L. (1981). Seat belt use and condition and driver attitudes. Helsinki: Central Organization for Traffic Safety.

Pace, B. J., Thailer, R., \& Kwiatkowski, T. G. (1986). New York State mandatory seatbelt use law: Patterns 
of seatbelt use before and after legislation. The Journal of Trauma, 26, 1031-1033.

Partyka, S. (1987, February). Mandatory belt use effects in 1985. In Research notes. Washington, DC: U.S. Department of Transportation, NHTSA, National Center for Statistics and Analysis.

Pierce, J. A. (1979). Safety benefits of the seat belt legislation and speed limit reduction in Ontario. In Proceedings of the 23rd Annual Conference of the American Association for Automotive Medicine (pp. 242253). Morton Grove, IL: American Association for Automotive Medicine.

Pye, G., \& Waters, E. A. (1984). Effect of seat belt legislation on injuries and road traffic accidents in Nottingham. Rritish Journal of Medicine, 288, 756757.

Road Traffic Board of South Australia. (1983). Seat belt usage before and after a multi-media advertising campaign, August-November, 1982. Walkerville, South Australia: Author.

Rood, D. H., Kraichy, P. P., \& Carubia, J. (1985). Evaluation of New York State's mandatory occupant restraint law: Vol. I. Observational surveys of safety restraint use in New York State. Albany: SUNY-Albany, Institute for Traffic Safety Management and Research. (NTIS No. DOT HS 806950)

Schnerring, F. (1983). Surveys of seat belt wearing in New South Wales: 1970-1981. New South Wales: Traffic Accident Research Unit, Traffic Authority of New South Wales.

Seeney, K. M. (1977). Queensland experience of compulsory wearing of seat belts. In Proceedings of the Sixth International Conference of the International Association for Accident and Traffic Medicine (pp. 115-124). Stockholm: International Association for Accident and Traffic Medicine.

Shiels, A. C. (1978, October). The effects of seat belt legislation: The Saskatchewan experience. Paper presented at the 10th Annual Safety Conference of the Canada Safety Council, Winnipeg.

Simpson, H. M., \& Warren, R. A. (1981). Seat belts and traffic safely: The Canadian experience. In R. Green \& E. Petrucelli (Eds.), The human collision: Proceedings of the International Symposium on $\mathrm{OC}$ cupant Restraints (pp. 73-83). Morton Grove, IL: American Association for Automotive Medicine.

Snow, J. W. (1979). Seat belt legislation in Ontario,
Canada: Its history and effects. In '79 International Symposium on Seat Belts in Tokyo (pp. 199-202). Tokyo: Japanese Council of Traffic Science.

Stulginskas, J. V., \& Pless, I. B. (1983). Effects of a seat belt law on child restraint use. American Journal of Disabled Children, 137, 582-585.

Toomath, J. B. (1977). Compulsory seat belt use in New Zealand. In Proceedings of the Sixth International Conference of the International Association for Accident and Traffic Medicine (pp. 21-39). Stockholm: International Association for Accident and Traffic Medicine.

Trinca, G. W. (1984). Thirteen years of seat belt useHow great the benefits. In Advances in belt restraint systems: Design, performance, and usage (pp. 1-5) Warrendale, PA: Society of Automotive Engineers.

Trinca, G. W., \& Dooley, B. J. (1977). The effects of seat belt legislation on road traffic injuries. Australia and New Zealand Journal of Surgery, 472, 150-155.

Vaaje, T. (1986). Safety belt usage in various countries. In Effectiveness of safety belt use laws: A multinational examination (pp. 13-23). Washington, DC: NHTSA. (NTIS No. DOT HS 807018)

Vulcan, A. P. (1977). Victorian experience with the compulsory wearing of seat belts. In Proceedings of the Sixth International Conference of the International Association for Accident and Traffic Medicine (pp. 99-104). Stockholm: International Association for Accident and Traffic Medicine.

Wagenaar, A. C., Maybee, \& Sullivan, D. P. (1987). Michigan's compulsory restraint use policies: Effects on injuries and deaths. Ann Arbor: The University of Michigan Transportation Research Institute.

Wagenaar, A. C., Molnar, L. J., \& Businski, K. L. (1987). Direst observation of seat helt use in Michigan: December 1986. Ann Arbor: The University of Michigan Transportation Research Institute.

Wagenaar, A. C., \& Webster, D. (1986). Preventing injuries to children through compulsory automobile safety seat use. Pediatrics, 78 662-672.

Williams, A. F., Preusser, D. F., Blumberg, R. D., \& Lund, A. K. (in press). Results of a seat belt use law enforcement and publicity campaign in Elmira, New York. Accident Analysis and Prevention.

Williams, A. F., Wells, J. K., \& Lund, A. K. (1986). Shoulder belt use in four states with belt use laws. Washington, DC: IIHS.

\section{APPENDIX}

\section{Autoregressive Integrated Moving Average/Transfer Function Model}

$$
\begin{aligned}
& \left(1-\phi_{1} B-\ldots \phi_{p} B^{p}\right)\left(1-\Phi_{1}, B-\ldots \Phi_{P} B^{P_{s}}\right)(1-B)^{d}\left(1-B^{s}\right)^{D} \operatorname{Ln} Y_{t}=\alpha+\left(1-\theta_{l} B-\ldots \theta_{q} B^{q}\right)\left(1-\Theta_{l} B^{s}\right. \\
& \left.-\ldots \Theta_{Q} B^{Q s}\right) u_{t}+\psi X_{t}+\omega I_{t},
\end{aligned}
$$

$$
\begin{aligned}
& B=\text { the backshift operator such that } B\left(z_{t}\right) \\
& \text { equals } z_{t-1}
\end{aligned}
$$

$$
\emptyset_{1} \text { to } \varnothing_{p}=\text { regular autoregressive parameters }
$$

$q=$ order of the moving average process

$\theta_{l}$ to $\theta_{q}=$ regular moving average parameters 
$\Phi_{1}$ to $\Phi_{p}=$ seasonal autoregressive parameters

$d=$ order of nonseasonal differencing

$D=$ order of seasonal differencing

$s=$ seasonal span

$\operatorname{Ln} Y_{3}=$ natural logarithm transformation of the dependent time series

$\alpha=a$ constant
$\Theta_{1}$ to $\Theta_{Q}=$ seasonal moving average parameters

$u_{t}=$ random error component

$\psi$ and $\omega=$ intervention parameters to be estimated

$X_{t}=$ step function with the value 1 beginning at month $t$ and 0 otherwise

$I_{t}=$ step function with the value 1 beginning at month $t$ and 0 otherwise

Final statistical models for selected variables are included here. Standard errors are shown in parentheses below each parameter estimate.

Aggregate Relative Rate of Front-seat Fatalities Age 10 and Over per VMT for Eight Belt-law versus Eight Comparison States

$(1-B) \operatorname{Ln} Y_{\mathrm{t}}=\left(1-.857 B-.089 B^{3}\right) u_{\mathrm{t}}-(1-B) .098 X_{79}-(1-B) .091 I_{108}$

Adjusted $\mathrm{R}^{2}=.51$

$$
\begin{array}{lll}
(.075)(.079) & (.028) & (.034)
\end{array}
$$

Aggregate Rate of Rear-seat Fatalities Age 10 and Over per VMT for Eight Belt-law States

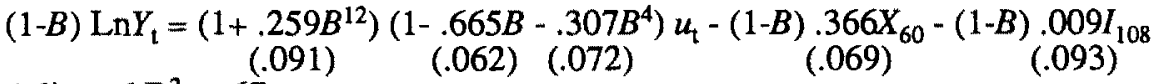

Adjusted $\mathrm{R}^{2}=.67$

Aggregate Rate of Nonoccupant Fatalities Age 10 and Over per VMT for Eight Belt-law States

$(1-B)\left(1-B^{12}\right)\left(1+.551 B^{12}\right)(1+.347 B) \operatorname{Ln} Y_{1}=u_{1}-(1-B)\left(1-B^{12}\right) .062 X_{62}+(1-B)\left(1-B^{12}\right) .090 I_{108}$

Adjusted $\mathrm{R}^{2}=.87$

$$
(.081) \quad(.093)
$$

Aggregate Rate of Front-seat Fatalities Age 10 and Over per VMT for Two Primary Enforcement Provision States

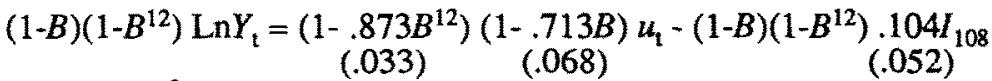

Adjusted $\mathrm{R}^{2}=.81$

$$
\text { (.033) }
$$

\section{Aggregate Rate of Front-seat Fatalities Age 10 and Over per VMT for Four Secondary} Enforcement Provision States

$\left(1-B^{12}\right) \operatorname{Ln} Y_{\mathrm{t}}=(1+.228 B) u_{\mathrm{t}}-\left(1-B^{12}\right) .144 X_{66}-\left(1-B^{12}\right) .070 I_{108}$
$(.097)$

Adjusted $\mathrm{R}^{2}=.69$

$$
(.097)
$$

